\title{
The Role of Mental Health Service Research in Promoting Effective Treatment for Adults with Schizophrenia $\dagger$
}

\author{
Anthony F. Lehman* \\ University of Maryland, Baltimore, Center for Mental Health Services Research, Baltimore, MD, USA
}

\begin{abstract}
Background: Significant gaps exist between scientific knowledge about the efficacy of treatments for mental disorders and the availability of efficacious treatments in routine practice. Mental health service research can help bridge this gap between basic clinical research and the usual care afforded adults with mental disorders.
\end{abstract}

Aims: To illustrate this potential, data on the efficacy of treatment for schizophrenia are reviewed.

Methods: The treatments reviewed include pharmacotherapies, psychological interventions, family interventions, vocational rehabilitation and assertive community treatment and case management. Using treatment recommendations based upon outcome data about these treatments and the results of a large survey of usual care for schizophrenia from the Schizophrenia Patient Outcomes Research Team (PORT) project, examples of current deficiencies in the usual treatment of adult mental disorders and relevant questions that need to be addressed by mental health services research are identified.

Results: Major deficiencies in treatment that were identified include inappropriate dosing with antipsychotic agents, underutilization of adjunctive antidepressant therapy, very low rates of prescription of psychosocial interventions and lack of continuity between inpatient and outpatient settings.

Discussion: These findings raise serious concerns about access to care and the appropriateness and quality of care that is offered.

Implications: This knowledge about what treatments work for schizophrenia and the patterns of current care suggest the following major questions be addressed by mental health services research: What is the nature of care currently being offered adults with mental disorders? To what degree does this care measure up to scientifically derived quality of care and treatment standards? What is the effectiveness of new technologies under usual practice conditions? For which patients are they cost-effective and under what conditions? How should financial incentives be structured within systems of care to promote the most cost-effective use of new technologies? How should service systems themselves be organized to promote appropriate access and utilization? What educational, organizational and financing interventions promote adoption of effective interventions? Do we have valid methods for assessing quality of care? What strategies (interventions) are effective at improving the quality of care? In addition, we need to develop strategies that transfer mental health services research technologies into practice. These include: (i) development of outcome measures that meet scientific standards and that are practical for general application in service systems to facilitate 'outcome management'; (ii) development of quality of care assessment methodologies that are practical and scientifically sound and (iii) cost-effectiveness methodologies.

Mental health services research can facilitate the translation of knowledge developed from basic clinical research into more effective systems of care. The tools used by health services research to this end include examination of patterns of usual care in relation to scientifically established standards of efficacious care, interventions to improve the effectiveness of care and examination of the impacts of the organization and financing of services on outcomes and costs. In short, mental health service research holds high on its agenda the translation of basic and clinical research into practice.

All of us must face the challenges posed by our rapidly changing mental health care system, changes driven not only by managed care and cost containment, but by exciting new developments in the treatment of mental disorders. We take on these challenges as researchers, clinicians, administrators, patients, families and taxpayers. Here I seek to provide a perspective on what we know about the treatment of adults with mental disorders and to discuss the implications of this knowledge for the work of mental health service research. Each of us has a particular window on this scene; mine is primarily that of a clinical mental health services researcher who studies schizophrenia. I will briefly summarize current knowledge about the efficacy of treatments for schizophrenia and the services research questions that this knowledge raises in its translation to clinical practice. The lessons from this examination readily generalize to the treatment of other adult mental disorders. (C) 1998 John Wiley \& Sons, Ltd.

Received 10 August 1998; accepted 6 October 1998.

*Correspondence to: Anthony F. Lehman, Center for Mental Health Services Research, University of Maryland at Baltimore, 685 West Baltimore Street, MSTE Building, Room 300, Baltimore, MD 21201-1549, USA $\dagger$ A version of this paper was presented at the NIMH Mental Health Services Research Conference in Washington, DC, on 5 September 1997. Source of funding: none declared. 


\section{Outcomes of Interventions for Schizophrenia: The Evidence}

\section{Pharmacotherapies}

Pharmacotherapies are the most extensively evaluated type of intervention for schizophrenia and include the socalled 'conventional' or older antipsychotic agents, such as chlorpromazine, haloperidol, fluphenazine and molindone, the newer antipsychotic agents, including clozapine, risperidone, olanzapine, quetiapine (Seroquel), sertindole and ziprasidone, and a variety of adjunctive agents, including anxiolytics, antidepressants and mood stabilizers. The antipsychotic agents are used to treat the psychotic symptoms of schizophrenia, whereas the adjunctive agents are prescribed primarily for related depression, anxiety, agitation and aggression or mood instability.

The evidence for the efficacy of conventional antipsychotic agents for reducing positive symptoms and symptom relapse is impressive. ${ }^{1}$ In nearly 100 randomized clinical trials, conventional antipsychotic agents were more efficacious versus placebo in reducing acute psychotic symptoms. Combined results of these studies show that $75 \%$ of patients improved on conventional antipsychotic medications compared to only $25 \%$ of placebo-treated patients. Similarly in nearly 50 randomized clinical trials of conventional antipsychotic agents versus placebo, annual relapse rates on the medications were about $20 \%$ compared to $55 \%$ for placebo-treated patients. ${ }^{1}$ Hence conventional antipsychotic medications substantially enhance symptom control. There is little evidence that these agents improve other outcomes.

There is also relatively substantial evidence on the preferred dosing strategies for using conventional antipsychotic medications. A variety of alternative strategies have been studied, including 'rapid neuroleptization', very high doses, intermittent dosing and very low doses. The net conclusion from these various studies is that moderate level doses, in the range of 300-1000 chlorpromazine (CPZ) equivalents daily for acute episodes and 300-600 CPZ equivalents daily for maintenance, are more efficacious than other approaches. Very low and intermittent dose strategies substantially increase the risk of relapse, and rapid and high dose strategies substantially increase the risk of side effects without offering additional efficacy. ${ }^{1}$

The efficacy data on the newer antipsychotic medications are more limited, but there is good evidence that they are as effective as the older agents in reducing positive symptoms. $^{2-7}$ The newer agents may also offer certain important advantages. Clozapine, for example, is known to have superior efficacy for reducing positive symptoms in about $30 \%$ of patients who fail to respond adequately to conventional agents. Its side effect profile, including a risk of agranulocytosis, however, limits its use to patients who fail to respond to or cannot tolerate other antipsychotic agents. The other newer agents currently on the US market are risperidone, olanzapine and Seroquel. These agents offer first-line alternatives to the older agents because they produce fewer extrapyramidal side effects at therapeutic dose levels. It remains an area of continued inquiry whether these newer agents have additional efficacy for symptoms other than the positive symptoms. Clozapine appears to favorably affect depression and anxiety and perhaps the negative symptoms of schizophrenia. Data are less conclusive in this regard for olanzapine, risperidone and Seroquel. The effects of sertindole and ziprasidone are not mentioned here because they are not yet on the US market, and data are more limited. Overall there is hope that the newer antipsychotic agents will offer better treatment effectiveness that the older agents, perhaps through direct effects on negative symptoms, depression, anxiety, hostility and less problematic side effect profiles. ${ }^{8}$

There are few data to suggest that antipsychotic agents have substantial impacts on the functional disabilities that accompany schizophrenia. Whether the newer agents are more efficacious in this regard remains uncertain. Limited data indicate that subjective quality of life experiences are related to medication side effects and secondary mood disturbances. $^{8}$ The newer agents may offer advantages on these outcome dimensions.

Beyond the antipsychotic agents, a number of other types of psychotropic agent may be useful in the treatment of schizophrenia. There are relatively good data to indicate that antidepressants should be prescribed to patients who experience persistent depression after apparently adequate treatment of the psychotic symptoms. ${ }^{9}$ Similarly patients with persistent anxiety or agitation may benefit from adjunctive anxiolytic therapy. ${ }^{9}$ Failure to treat these symptoms may result in poorer outcomes.

\section{Psychological Interventions}

The efficacy literature on psychological interventions is far more limited than that for pharmacotherapies. ${ }^{10}$ Psychological interventions that have been studied include various models, individual and group psychotherapies as well as skills training. Within the limitations of the existing studies, it can be stated that supportive, reality-based individual and group therapies that focus on practical life problems associated with the illness have proven more effective than psychodynamically oriented therapies. Furthermore there are some concerns that psychoanalytically oriented therapies promoting regression are potentially harmful to patients with schizophrenia. 'Supportive' psychotherapies have widespread appeal and use, but often are not well defined. There is considerable need for research on the value of supportive therapy in the care of persons with schizophrenia. Interest is growing in cognitive-behavioral therapies in schizophrenia, but research is still under way on these. Individual and group therapies that employ well specified combinations of support, education and behavioral and cognitive skills training designed to address the specific deficits and challenges posed by schizophrenia should be offered to improve functioning and adaptation to the illness. These treatments, of course, should be offered in conjunction with pharmacotherapy. 


\section{Family Interventions}

Interventions that educate families about schizophrenia, provide support and offer training in effective problemsolving and communication have been subjected to numerous randomized clinical trials. ${ }^{11}$ The data strongly and consistently support the value of such interventions in reducing symptom relapses, and there is some evidence that they contribute to improved patient functioning and family wellbeing. These randomized clinical trials have reported one year relapse rates in the range of $2-23 \%$ for patients receiving these family 'psychoeducation' programs in combination with pharmacotherapy compared to relapse rates of 40-53\% with pharmacotherapy alone. This relative reduction in relapse rates persisted for at least two years in the one clinical trial that followed patients for that long. ${ }^{12}$ A recent study found psychoeducation programs using multiple family groups to be more effective and less expensive than individual family psychoeducational interventions. ${ }^{13}$ The net conclusion from the research on family psychoeducational interventions is that these should be offered to all patients with schizophrenia who have some on-going contacts with their families.

\section{Vocational Rehabilitation}

Work is widely viewed as an important goal in the treatment and rehabilitation of persons with schizophrenia. Work in this context has been viewed both as a therapeutic modality, generating a sense of purpose and self-esteem, and as an end in itself, allowing a person to achieve personal independence and reducing the economic burden on society. However, controlled studies of vocational rehabilitation interventions have not shown consistent or significant impacts on outcomes. ${ }^{14}$ While it is true that such programs increased vocational activities while patients participated in them, there is little evidence that they enhance work performance or achievement outside of the program. On a more encouraging note, more recent controlled studies have reported significantly improved vocational outcomes for an individualized employment program, which emphasizes rapid placement in a real job setting and strong supports from an employment specialist to adapt and sustain the job. ${ }^{14}$ More research is needed to confirm these initially promising results.

\section{Assertive Community Treatment and Case Management}

A final area of intervention that has received considerable research attention is case management and assertive community treatment. These are actually substantially different types of intervention, but are grouped together here because they are often discussed together elsewhere and even erroneously equated. Case management refers to a range of strategies for enhancing continuity of care and functioning in the community. There are several models of case management. The broker model emphasizes advocacy and linkage of the patient with existing community services.
The broker case manager provides no direct services other than these intermediary services. The clinical case management model incorporates the advocacy and linkage of the broker model, but emphasizes the clinical relationship between the patient and the case manager, who functions in a therapeutic mode with the patient as well. The rehabilitation and strength based models also incorporate the broker functions, but emphasize a rehabilitation approach, focusing on functional improvements and building upon the patients' existing functional assets. Research on these various models has failed to produce substantial evidence on efficacy although it can generally be stated that models that focus on specific outcomes, for example, enhancing the patient's ability to live in a particular community environment, are more effective than more global models with less clear goals. ${ }^{15}$

The Program of Assertive Community Treatment (PACT) is a specific model of community based care. Originating with an experiment in Madison, WI, in the late 1970s, PACT moved the multidisciplinary inpatient team of the state hospital into the community. The team took with it all of the functions of an inpatient team: interdisciplinary team work, 24 hour/7 days per week coverage, comprehensive treatment planning, on-going responsibility, staff continuity and small caseloads. PACT is designed to treat patients who are at high risk for hospital readmission and who cannot be maintained by more usual community based treatment. Randomized trials comparing PACT to other community-based care have consistently shown that PACT substantially reduces inpatient utilization and promotes continuity of outpatient care. ${ }^{16}$ Patient satisfaction with this model is generally high, and family advocacy groups, such as the National Alliance for the Mentally Ill in the United States, strongly support its use and dissemination. Results are less consistent on the impacts of PACT on other outcomes although at least some studies have shown enhancement of clinical status, functioning and quality of life. Cost-effectiveness studies support its value in high-risk cases. Studies also indicate that a particular PACT program's effectiveness is related to the fidelity with which it is implemented, that is, the degree to which the program adheres to the original PACT model, as described above.

\section{Translating Research into Practice}

Translating scientific knowledge about treatment efficacy into routine quality care remains a major challenge for all of medicine. ${ }^{17}$ One recent extension of clinical research has been the development of 'disease management' strategies, typified by 'practice guidelines' and 'treatment algorithms'. A variety of empirically based guidelines for the treatment of schizophrenia have recently become available. These include the Schizophrenia PORT Treatment Recommendations, ${ }^{18}$ the American Psychiatric Association Schizophrenia Practice Guidelines, ${ }^{19}$ a set of recently published expert consensus guidelines ${ }^{20}$ and expert consensus guidelines for inpatient treatment of schizophrenia. ${ }^{21}$ The concurrent introduction of all of these demonstrates both the demand 
for such guidelines as well as the range of methods used to generate them.

Disease management in schizophrenia lags substantially behind other disorders for a variety of reasons. First, the chronicity and enduring disabilities associated with schizophrenia make the challenges of developing disease management standards more difficult than for less chronically disabling conditions. ${ }^{22}$ Treatment for persons with schizophrenia must address a variety of social needs related to disability (housing, income support and unemployment) in addition to clinical needs (symptom reduction). Second, the care of persons with schizophrenia occurs largely in the public mental health specialty sector and is paid for largely by government insurance or entitlement programs (Medicaid, Medicare, VA). ${ }^{23,24}$ This has placed treatment for schizophrenia on a lower priority for managed care, a priority that is rapidly changing with the advent of managed care for Medicaid and Medicare recipients. Third, as mentioned previously, it is only now that empirically based standards of care for schizophrenia are becoming available. Fourth, collection of data on outcomes in routine practice from persons with schizophrenia may pose greater challenges than for other diagnostic groups because of the complexity of the outcomes ${ }^{22}$ and concerns about the validity of outcome data collected from persons with this disorder. A variety of outcome measures are now or are about to become available that make collection of outcome information on persons with schizophrenia much more efficient and feasible for large systems of care. ${ }^{25,26}$

\section{Some Lessons from the Schizophrenia PORT}

I will use the Schizophrenia PORT Recommendations to illustrate the issues raised by such disease management innovations. Treatment of serious mental disorders has been hobbled over the years by the lack of clear standards of care. This lack of standards has made it difficult to hold practitioners, service systems and payers accountable. In the absence of definitive standards, individual 'expert opinion' and cost containment prevail, even when contrary to the scientific evidence on what works. Scientifically based quality of care standards promise to change this.

The Schizophrenia PORT project recently surveyed over 700 persons under treatment for schizophrenia to determine the degree to which their treatment met standards defined by the PORT Treatment Recommendations. ${ }^{27}$ The general conclusions from this survey were the following.

(i) For nearly all of the recommendations the level of conformance was modest at best. For all but one recommendation, fewer than half of the patients were receiving treatment that met the recommendation criteria.

(ii) Overall, rates of conformance were lower for the psychosocial treatment recommendations than for the pharmacological recommendations.

(iii) Few consistent relationships were found between conformance with the recommendations and patient demographics. Younger patients were more likely to be offered psychotherapy and vocational rehabilitation. Of concern is the finding that African-American patients were more likely to be on higher doses of antipsychotic medications and to be prescribed antiparkinson agents more often for side effects. African-American patients also were less likely to be prescribed an antidepressant medication when depressed.

(iv) The treatment plans suggest low rates of use of psychosocial treatments. Failure to consider these important treatments for more stable outpatients may be a serious problem in on-going communitybased care.

(v) Patterns of recommendation conformance varied by location for the psychosocial treatments, but much less so for the pharmacological recommendations. The appropriate use of psychosocial interventions may be more vulnerable to local idiosyncrasies than the pharmacological treatments.

(vi) The treatment of patients in rural areas was more consistent with the recommendations than was that of urban patients.

To illustrate both the health care delivery and health services research challenges raised by the PORT, I will consider a few of the PORT Treatment Recommendations. Specifically the following recommendations will be examined:

Recommendation 1. Antipsychotic medications, other than clozapine, should be used as the first-line treatment to reduce psychotic symptoms for persons experiencing an acute symptom episode of schizophrenia.

Recommendation 9. The maintenance dosage should be in the range of 300-600 CPZ equivalents (oral or depot) per day.

Recommendation 13. A trial of clozapine should be offered to patients with schizophrenia and schizoaffective disorder whose positive symptoms do not respond robustly to trials of two different types of conventional antipsychotic medication.

Recommendation 23. Individual and group therapies employing well specified combinations of support, education, behavioral, cognitive and skills training approaches designed to address the specific deficits of persons with schizophrenia may be useful over time to improve functioning and enhance other targeted problems, such as medication non-compliance.

Recommendation 24. Patients who have on-going contact with their families should be offered a family psychosocial intervention which spans at least nine months and which provides a combination of education about the illness, family support, crisis intervention and problem solving skills training. Such interventions should also be offered to non-family caregivers. 
Recommendation 27. Persons with schizophrenia who have any of the following characteristics should be offered vocational services. The person: (i) identifies competitive employment as a personal goal; (ii) has a history of prior competitive employment; (iii) has a minimal history of psychiatric hospitalization; (iv) is judged on the basis of a formal vocational assessment to have good work skills.

Recommendation 29. Systems of care serving persons with schizophrenia who are high service utilizers should include assertive case management and assertive community treatment programs.

The first issue raised is access to treatments. All of the PORT Recommendations have implications for access, but the specifics vary depending upon the recommendation. Recommendation 1 appears at first glance straightforward; that is, that psychosis should be treated with antipsychotic medications. However, an important aspect of this recommendation is that it includes access to the newer antipsychotic medications as first-line agents. Many treatment systems currently choose to restrict first-line access to these drugs because of costs. A typical daily dose of an older, generic antipsychotic medication, haloperidol, costs about $\$ 0.10$; a typical daily dose of one of the new agents, risperidone, costs about $\$ 10$. Such cost differentials create substantial questions about cost-effectiveness, cost-offsets and the value of these new technologies. Similarly, recommendation 13 states the need for access to clozapine for treatmentrefractory patients. While most treatment systems currently provide some access to clozapine, this is far from uniform across treatment systems. Even within a treatment system, clozapine may be available within only certain components. For example, PORT interviews with care providers revealed lack of continuity of access between inpatient and outpatient settings. In some cases, the new agents could only be initiated in a state hospital. The logic of such a restriction is to ensure that only the patients most in need receive this expensive treatment. An unintended effect is the incentive to admit a patient to a state hospital in order to gain access to clozapine and the other new agents. In other cases, the costs for the new agents were covered by the state only while the patient is in the hospital. This creates a catch-22 for patients who respond to the newer agents while in the hospital, but who cannot afford them after discharge. Consumer and provider acceptance of these new pharmacotherapies is not a major barrier. Indeed consumer advocates, families and practitioners generally insist that these medications should be available. The issue is whether service systems and payers will respond to this pressure based upon their assessments of cost-effectiveness.

The recommendations on psychosocial treatments (for example, recommendations 23, 24, 27 and 29) raise additional concerns about access. The PORT survey found that few patients receive these psychosocial interventions despite the substantial evidence that adding psychosocial interventions to medications enhances outcomes in schizophrenia. The PORT found low rates of access to any form of psychotherapy
(45\%), family psychoeducation programs (10\%) and vocational rehabilitation $(23 \%)$. The health services issues associated with these psychosocial technologies differ from those associated with the new antipsychotic agents. While cost is a barrier to these as well, there are additional barriers due to provider knowledge and acceptance. In contrast to new medications, new psychosocial treatments require extensive clinician training and often a theoretical shift in the clinician's orientation to the problem. For example, adoption of the family psychoeducation interventions requires viewing families as collaborators in treatment of the patient, rather than patients themselves. These family interventions also require changes in agency logistics and procedures. The multiple family psychoeducation program of McFarlane ${ }^{13}$ involves some shifts from individual patient sessions to multiple family meetings, operating hours that are convenient to working families and the capacity to bill for this type of service. All of these can be barriers to implementing this cost-effective technology.

A second issue raised by the PORT is the appropriateness and quality of the interventions offered. Treatments may be accessible within a system of care, but may not be prescribed or implemented appropriately. Recommendation 9 specifies the usual dosage range of maintenance antipsychotic therapy based on many clinical trials. The PORT found that only $29 \%$ of patients were prescribed dosages in this range and that African-Americans were more likely to be prescribed higher doses than Whites. Similarly, African-Americans with schizophrenia and co-morbid depression were less likely to receive an adjunctive antidepressant than were Whites with similar co-morbidity. Such patterns illustrate problems of appropriateness and quality of treatment even when access is not a problem. Similar issues arise in the offering of psychosocial treatments. The PORT was not able to determine the degree to which 'psychotherapy', 'family therapy', 'vocational rehabilitation' and 'assertive community treatment' prescribed in medical records conformed to the models developed and evaluated in clinical trials. However, based upon our interviews with agencies that participated in the PORT, it seems likely that often these treatments are not of adequate quality. For example, caseloads for so-called assertive community treatment teams typically substantially exceeded those specified by the model. Few, if any, programs offered formal family psychoeducation programs or the newer models of individualized vocational rehabilitation. Most psychotherapy was non-specific.

\section{The Mental Health Services Research Agenda}

Although the PORT project illustrates some answers about the current state of treatment for schizophrenia, this knowledge about what treatments work for schizophrenia and the patterns of current care suggest the following major questions be addressed in an ongoing way by mental health services research. What is the nature of care currently being offered adults with mental disorders and how is it changing over time? To what degree does this care measure up to 
scientifically derived quality of care and treatment standards? What is the effectiveness of new technologies under usual practice conditions? For which patients are they costeffective and under what conditions? How should financial incentives be structured within systems of care to promote the most cost-effective use of new technologies? How should service systems themselves be organized to promote appropriate access and utilization? What are the workforce barriers to appropriate implementation of interventions? What are the organization and financing barriers to implementation of these interventions? What educational, organizational and financing interventions promote adoption of effective interventions? Do we have valid methods for assessing quality of care? Does efficacy translate into effectiveness? Do quality of care criteria predict better outcomes? What strategies (interventions) are effective at improving the quality of care? Interventions with providers, consumers, programs, service systems, purchasers? What is the evolving interfaces between the mental health system with other service sectors?

In addition, we need to develop strategies that transfer mental health services research technologies into practice. These include

(i) development of outcome measures that meet scientific standards and that are practical for general application in service systems to facilitate 'outcomes management',

(ii) development of quality of care assessment methodologies that are practical and scientifically sound and (iii) cost-effectiveness methodologies.

The critical issue here is whether the types of method used in externally funded research can be adapted for application to the 'real world'. While such applications will not take the place of rigorous, externally funded research, they are sorely needed to ensure that knowledge developed in the mental health services research laboratory is translated into practice.

\section{References}

1. Dixon L, Lehman A, Levine J. Conventional antipsychotic medications for schizophrenia. Schiz. Bull. 1995; 21: 567-578.

2. Beasley CM, Tollefson GD, Tran P, Satterlee W, Sanger T, Hamilton S. Olanzapine versus placebo and haloperidol: acute phase results of the North American double-blind olanzapine trial. Neuropsychopharmacology 1996; 14: 105-110.

3. Marder SR, Meibach RC. Risperidone in the treatment of schizophrenia. Am. J. Psychiatry 1994; 151: 825-835.

4. Kane J, Honigfeld G, Singer J, Meltzer HY, and the Clozaril Collaborative Study. Clozapine for the treatment-resistant schizophrenic: a double-blind comparison with chlorpromazine. Arch. Gen. Psychiatry 1988; 45: 789-796.
5. Borison RL, Arvantis LA, Miller BG. A comparison of five fixeddoses of 'Seroquel' (ICI-204,636) with haloperidol and placebo in patients with schizophrenia. Schiz. Res. 1996; 18: 132

6. Schulz SC, Mack R, Zborowski J, Morris D, Sebree T, Wallin B. Efficacy, safety, and dose response of three doses of sertindole and three doses of Haldol in schizophrenia patients. Schiz. Res. 1996; 18: 133.

7. Seeger TF, Seymour PA, Schmidt AW. Ziprasidone (CP-88, 059): a new antipsychotic with combined dopamine and serotonin receptor antagonist activity. J. Pharm. Exp. Ther. 1995; 275: 101-113.

8. Lehman AF. Evaluating outcomes of treatments for persons with psychotic disorders. J. Clin. Psychiatry 1996; 57 (suppl 11): 61-67.

9. Johns C, Thompson J. Adjunctive treatments in schizophrenia: pharmacotherapies and electroconvulsive therapy. Schiz. Bull. 1995; 21: $607-619$.

10. Scott J, Dixon L. Psychological interventions for schizophrenia. Schiz. Bull. 1995; 21: 621-630.

11. Dixon L, Lehman A. Family interventions for schizophrenia. Schiz. Bull. 1995; 21: 631-643.

12. Hogarty GE, Anderson CM, Reiss DJ, Kornblith SJ, Greenwald DP, Ulrich RF, Carter M. Family psychoeducation, social skills training, and maintenance chemotherapy in the aftercare treatment of schizophrenia: two-year effects of a controlled study on replase and adjustment. Arch. Gen. Psychiatry 1991; 48: 340-347.

13. McFarlane WR, Lukens E, Link B, Dushay R, Deakins SA, Newmark M, Dunne EJ, Horen B, Toran J. Multiple family group and psychoeducation in the treatment of schizophrenia. Arch. Gen. Psychiatry 1995; 52: 679-687.

14. Lehman A. Vocational rehabilitation in schizophrenia. Schiz. Bull. 1995; 21: 645-656.

15. Attkisson C, Cook J, Karno M, Lehman AF, McGlashan T, Meltzer H, O'Connor M, Richardson D, Rosenblatt A, Wells K, Williams J, Hohmann A. Clinical services research. Schiz. Bull. 1992; 18: 561-626.

16. Scott J, Dixon L. Assertive community treatment and case management. Schiz. Bull. 1995; 21: 657-668.

17. Brook RH. Managed care is not the problem, quality is. J. Am. Med. Assoc. 1997; 278: 1612-1614.

18. Lehman AF, Steinwachs DS and the Schizophrenia PORT CoInvestigators. Translating research in to practice: the Schizophrenia PORT treatment recommendations. Schiz. Bull. 1998; 24: 1-10.

19. American Psychiatric Association. Practice guideline for treatmen of patients with schizophrenia. American Psychiatric Association Press, 1997.

20. Frances A, Docherty J, Kahn DA. The expert consensus guideline series: treatment of schizophrenia. J. Clin. Psychiatry 1996; 57 (suppl 12B): 1-58.

21. McGlynn E. Inpatient treatment of schizophrenia: a literature review and criteria for assessing quality. RAND monograph DRU-451NIMH, 1993.

22. Lehman AF. Measuring quality of life in a reformed health system Health Affairs 1995; 14: 90-101.

23. Norquist GS, Regier DA, Rupp A. Estimates of the cost of treating people with schizophrenia: contributions of data from epidemiologic surveys. In: Moscarelli M et al., eds. Handbook of Mental Health Economics and Health Policy. Vol. 1: Schizophrenia. New York: Wiley, 1996, 95-101.

24. Rupp A, Keith S. The costs of schizophrenia: assessing the burden. Psych. Clin. N. Am. 1993; 16: 413-423.

25. Sederer L, Dickey B (eds). Outcomes assessment in clinical practice. Baltimore, MD: Williams and Wilkins, 1996.

26. Thornicroft G, Tansella M (eds). Mental health outcome measures. Berlin: Springer, 1996.

27. Lehman AF, Steinwachs DS and the Schizophrenia PORT CoInvestigators. Patterns of usual care for schizophrenia: initial results from the Schizophrenia PORT client survey. Schiz. Bull. 1998; 24: $11-20$. 